\title{
Un marco exploratorio para el emprendimiento desde una perspectiva evolutiva
}

\section{An exploratory framework for Entrepreneurship from an evolutionary perspective}

\begin{abstract}
Dr. Oscar Javier Montiel-Méndez es profesor e investigador en la Universidad Autónoma de Ciudad Juárez (México) (oscar.montiel@uacj.mx) (http://orcid.org/0000-0003-0434-1649)
\end{abstract}

Dra. Argentina Soto-Maciel es profesora e investigadora en la Universidad Anahuac (México) (argentina.soto@anahuac.mx) (http://orcid.org/0000-0003-2671-1479)

\begin{abstract}
Resumen
La investigación en emprendimiento está evolucionando más que nunca (Carlsson et al., 2013). Como una línea de investigación que está ganando cada vez más atención, es imperativo entenderla más profundamente, dada la relevancia del emprendimiento, el emprendedor y las empresas en la economía global actual y bajo una lente multidimensional. A través de una extensa revisión de la literatura, se realizó una visión exploratoria de su perspectiva evolutiva, tomando como punto de partida aquella darwiniana, donde se encontró la falta de un marco formal y, por tanto, se propone un modelo teórico tridimensional (Emprendimiento, Emprendedor y Empresa, EEE) para su estudio, donde el contexto multidimensional y la participación que generalmente tiene la familia influyen en las relaciones ida y vuelta e iterativas que aparecen entre las dimensiones y que están influenciadas por la perspectiva evolutiva. Los resultados sugieren que existe un gran interés en la academia en esta visión evolutiva, una necesidad de explorar más este enfoque, teórica y empíricamente bajo las relaciones que el modelo propone entre las diversas líneas de investigación en emprendimiento, empresa familiar, economía y teoría de la empresa, ecología de la población y la elección estratégica, así como en el propio emprendedor y en su cognición y aprendizaje emprendedor, para desarrollar estrategias y políticas públicas que apoyen a las empresas y al ecosistema emprendedor.
\end{abstract}

\begin{abstract}
Entrepreneurship research is evolving more than ever (Carlsson et al., 2013). As a research stream that is gaining more and more attention, it is imperative to understand it more deeply, given the relevance of entrepreneurship, entrepreneurship, and businesses in today's global economy, and under a multidimensional lens. Through an extensive literature review, an exploratory view of its evolutionary perspective was made, from a Darwinian initial point, where the lack of a formal framework was found and therefore a 3-dimensional theoretical model (Entrepreneurship, Entrepreneur and Firm, EEF) is proposed for its study, where the multidimensional context and the involvement that the family generally has, influence the back and forth and iterative relationships that appear between the dimensions, and that is influenced by the evolutionary perspective. The results suggest that there is great interest in the academic field in this evolutionary view, a need to further explore this approach, theoretically and empirically under the relationships the model proposed among the various lines of research in entrepreneurship, family business, economics, and firm theory, population ecology, and strategic choice, as well as in the entrepreneur himself and its entrepreneurial cognition and learning, to develop strategies and public policies that support the organizations and the various stakeholders of the entrepreneurial ecosystem.
\end{abstract}

\section{Palabras clave I keywords}

Evolución, emprendimiento, emprendedor, empresa, elección estratégica, ecología poblacional, ecosistema emprendedor, contexto.

Evolution, entrepreneurship, entrepreneur, firms, strategic choice, population ecology, entrepreneurial ecosystem, context.

Forma sugerida de citar: Montiel-Méndez O.J., y Soto-Maciel, A. (2020). Un marco exploratorio para el emprendimiento desde una perspectiva evolutiva. Retos Revista de Ciencias de la Administración y Economía, 10(20), pp. 361-373. https://doi.org/10.17163/ret.n20.2020.10 


\section{Introducción}

Según el ciclo de la invención de Tina Seelig, todo inicia desde la imaginación, la creatividad, la innovación, y el emprendimiento. Este es un círculo que se basa en la génesis y la dinámica de una empresa (sea cual sea su naturaleza, no sólo está centrado en los negocios).

Una vez que un hecho social de este tipo comienza, ¿cómo asegurar su evolución y evitar su fracaso? Es bien conocido que las pequeñas empresas duran poco en todas las economías contemporáneas. Esto se evidencia a lo largo de diversos estudios, así como en el alto impacto que estas empresas tienen en algunos indicadores nacionales, como por ejemplo el empleo.

Por lo tanto, resulta muy importante investigar y mencionar la dinámica de varias empresas de este sector. La socialización de estas experiencias les permite a estos sectores estar informados con diversos grupos de interés, como el gobierno y las instituciones educativas, en el llamado ecosistema emprendedor (Malecki, 2011; Jackson, 2011) lo que es vital, incluso cuando podría estar en discusión si este constructo existe o es sólo una alegoría biológica.

Debido a nuestra economía, pareciera ser bastante pretencioso afirmar que existen tales ecosistemas. Desde el momento en que se habla de una política de innovación, ¿existe un sistema de innovación? Incluso si no funciona (¿Es disfuncional en México, y en general, en América Latina)? Así como hay una tipología para las empresas (micro, pequeña, mediana, y grande), entonces tal vez debería haber una para los ecosistemas, y que, si no se hace más énfasis para categorizarla, se corre el riesgo que este concepto se pudiera prostituir de alguna forma. Por lo tanto, sería necesario categorizarla y, una vez que sea funcional, llamarlo un ecosistema. De esta forma, se propone llamarlo un sistema de innovación, y cuando sea funcional llamarlo ecosistema, ya que, al tratarse de sistemas dinámicos complejos, se energizan los flujos y las existencias de capacidades.

Los estudios sobre las empresas familiares muestran que la relación entre el emprendimiento y las pequeñas empresas resulta ser una preocupación constante, y se sugiere que haya una evolución convergente. En la biología evolutiva esta evolución tiene lugar cuando especies de diferentes ancestros comparten rasgos similares debido a un ambiente compartido u otra presión de selección. Las ballenas y los peces tienen características similares, ambos desarrollaron métodos para moverse en el mismo medio: el agua. En el caso de estudio es el mercado.

Por lo tanto, es necesario garantizar la evolución de estas empresas hacia empresas consolidadas y establecidas para cualquier política pública relacionada con el tema. Ya no es posible seguir permitiendo errores, porque no sólo, como se mencionó, son aquellos primeros años en los que la organización lucha por sobrevivir ¿Y qué hay de la evolución (¿involución?) presentada por estos y el propio empresario a medida que pasan los años? ¿Qué sucede cuando el emprendedor y la organización entran a una zona de confort?

Varios factores pueden crear un terreno fértil para esto. Este es el problema de la sucesión, la falta de innovación, el altruismo, el dilema del samaritano, el lado oscuro de las empresas familiares. Sin olvidar la multidimensionalidad, la creación de una teoría basada en contribuciones de la evolución social que puede abordar los diversos vínculos y efectos entre el emprendimiento, el emprendedor y las pequeñas empresas, 
pero también como preventivo de un futuro fracaso. Millones de puestos de trabajo lo exigen, al igual que las economías nacionales y la sociedad.

Hay una necesidad constante, casi obsesionada, de una visión cuantitativa sobre el emprendimiento, que trata de explicar y detectar los factores de influencia en el éxito de las empresas, el capital humano del emprendedor (educación, experiencia, control interno, necesidad de logro y resiliencia), recursos financieros, número de socios, frecuencia y amplitud de comunicación externa, olvidando de que todas estas variables necesitan evolucionar sólo por este simple hecho: los mercados, las organizaciones y las personas siempre están cambiando sus patrones. La evolución, una perspectiva evolutiva que podría proporcionar un marco poderoso para llevar los tres pilares (emprendimiento, emprendedor y empresa) a la teoría, precisamente porque es la actividad de introducir nuevas actividades, métodos de producción y productos en una economía, y la variación económica es el requisito previo para la transformación y el desarrollo económico, en donde el hecho histórico fundamental sobre el capitalismo es su capacidad interna de transformación (Metcalfe, 2006).

\section{Metodología}

Se realizó una extensa búsqueda de la literatura a través de múltiples bases de datos (por ejemplo, Google Scholar, Scopus, Springer, Proquest, etc). No se hizo una revisión sistemática ya que este enfoque evolutivo, como muestra la revisión de la literatura, es escaso en el campo del emprendimiento. Se utilizaron las palabras clave "Emprendimiento", Evolución, “Teoría” tanto en español como en inglés.

\section{Revisión de la literatura}

Hay que reconocer que la línea es hacia una visión más conservadora. Por ejemplo, Freeman (1991) debate en el uso de analogías biológicas en la teoría de la economía evolutiva, reconociendo al mismo tiempo el papel positivo de esas analogías en la estimulación de un enfoque histórico de los sistemas económicos y que los procesos selectivos desempeñan un papel importante en el desarrollo de la ciencia y la tecnología; sin embargo, indica que existen serios riesgos al usar la analogía biológica y afirmó que el entorno social no sólo es mucho más complejo que el medio natural, sino que el papel de la intervención es totalmente diferente en la evolución de la tecnología. Aldrich et al. (2008) comparte esta posición al reconocer que el propio Darwin sugirió la idea de generalizar los principios darwinianos principales para explicar la evolución de las entidades sociales; pero los autores también mencionan el riesgo de malentendidos, tergiversación, generalización, analogía o reduccionismo biológico de sus principios. Sin embargo, en la biología evolutiva, algunas especies "biológicamente locas" como la especie humana no están limitadas ni se autolimitan. La selección natural para la aptitud reproductiva eventualmente permite a todas las especies autolimitantes exitosas superar sus características autolimitantes; los más competentes hacen esto se forma inmediata, pero los menos competentes se diluyen a través de la extinción de la escasez darwiniana, persisten o tardan un poco más en extinguirse (Meredith, 1982). Por lo tanto, se emplea la noción de involución, en el sentido (estricto) de degradación, con repercusiones en términos de consecuencias cognitivas y prácticas (Wolff et al., 1999; Atran et al., 2004). 
Por lo tanto, los economistas y otros científicos sociales no tienen otra alternativa que abandonar los modelos de las ciencias naturales, ya sean mecánicas o biológicas, y desarrollar modelos apropiados para los sistemas que investigan. Uno de esos posibles enfoques es la noción del paradigma tecno-económico, pero Freeman olvida el punto de que incluso este paradigma está sujeto a evolución, ya que la tecnología, por definición, implica innovación, por lo que filosóficamente lo que propuso se contradice a sí mismo.

Sobre la base de la revisión de la literatura, se propone explorar la perspectiva evolutiva desde 3 dimensiones estrechamente relacionadas (emprendimiento, emprendedor y la empresa), que resulta ser una visión novedosa en la literatura.

\subsection{Dimensión 1: Emprendimiento}

Veciana (1999) ofrece una perspectiva (Figura 1) sobre los diversos enfoques teóricos del emprendimiento (evolutivo por naturaleza). Allí, en el nivel meso del análisis está la teoría evolutiva. Dos elementos parecen faltar en la tabla: la Teoría del caos (Bygrave \& Hofer, 1991) y la Teoría de la complejidad (Mitchell, 2009) aplicada en el Emprendimiento (Lichtenstein, 2000), Investigación en Innovación y Tecnología (Berger \& Kuckertz, 2016). Por definición, la teoría evolutiva debe estar en todos los niveles.

Tabla 1. Enfoques teóricos del emprendimiento

\begin{tabular}{|c|c|c|c|c|}
\hline $\begin{array}{l}\text { Enfoques } \\
\text { Nivel } \\
\text { de análisis }\end{array}$ & $\begin{array}{l}\text { Enfoque } \\
\text { económico }\end{array}$ & $\begin{array}{c}\text { Enfoque } \\
\text { psicológico }\end{array}$ & $\begin{array}{c}\text { Enfoque } \\
\text { sociocultural }\end{array}$ & $\begin{array}{l}\text { Enfoque de } \\
\text { gestión }\end{array}$ \\
\hline $\begin{array}{l}\text { Micro } \\
\text { (Nivel } \\
\text { individual). }\end{array}$ & $\begin{array}{l}\text { - Función } \\
\text { emprendedora } \\
\text { como cuarto } \\
\text { factor de pro- } \\
\text { ducción. } \\
\text { - Teoría del } \\
\text { beneficio empre- } \\
\text { sarial. }\end{array}$ & $\begin{array}{l}\text { - Teoría de } \\
\text { rasgos. } \\
\text { - Teoría psico- } \\
\text { dinámica. }\end{array}$ & $\begin{array}{l}\text { - Teoría de la mar- } \\
\text { ginación. } \\
\text { - Teoría de roles. } \\
\text { - Teoría de la red. }\end{array}$ & $\begin{array}{l}\text { - Teoría de la eficien- } \\
\text { cia x de Leibenstein. } \\
\text { - Teoría conductual } \\
\text { del emprendedor. } \\
\text { - Modos de creación } \\
\text { de nuevas empresas. } \\
\text { - Modos para con- } \\
\text { vertirse en empren- } \\
\text { dedor. }\end{array}$ \\
\hline $\begin{array}{l}\text { Meso } \\
\text { (nivel } \\
\text { corporativo). }\end{array}$ & $\begin{array}{l}\text { Teoría de costos } \\
\text { de transacción. }\end{array}$ & & $\begin{array}{l}\text { - Teoría de la red. } \\
\text { - Teoría de la incu- } \\
\text { badora. } \\
\text { - Teoría evolutiva. }\end{array}$ & $\begin{array}{l}\text { - Modo de nuevo éxi- } \\
\text { to y fracaso empre- } \\
\text { sarial. } \\
\text { - Emprendimiento } \\
\text { corporativo. }\end{array}$ \\
\hline $\begin{array}{l}\text { Macro } \\
\text { (nivel de país). }\end{array}$ & $\begin{array}{l}\text { - Teoría del de- } \\
\text { sarrollo econó- } \\
\text { mico de Schum- } \\
\text { peter. }\end{array}$ & $\begin{array}{l}\text { - Teoría de } \\
\text { emprendedo- } \\
\text { res de Kirz- } \\
\text { ner. }\end{array}$ & $\begin{array}{l}\text { - Teoría del desa- } \\
\text { rrollo económico } \\
\text { de Weber. } \\
\text { - Teoría del inter- } \\
\text { cambio social. } \\
\text { - Teoría ecológica } \\
\text { de las poblaciones. } \\
\text { - Teoría institucio- } \\
\text { nal. }\end{array}$ & \\
\hline
\end{tabular}

Fuente: Veciana (1995-1999), en Veciana (2007, p. 35). 
Esta visión evolutiva también es tácita, por ejemplo, cuando en el entorno empresarial los intermediarios financieros, como las empresas de capital de riesgo (CR) seleccionadas, deben invertir con startups como un proceso evolutivo imprescindible tanto desde una visión organizativa como estratégica (Madsen \& Servais, 1997), o cuando una empresa debe aplicar la tendencia de sostenibilidad de la economía actual (Moore \& Manring, 2008). El enfoque naturalista requiere que se traten explícitamente los enfoques asociados a cómo reaccionan los seres humanos a los estados de incertidumbre.

El emprendimiento está en constante evolución (Landstrom, 2020), ampliando constantemente su dominio (Su, 2020; Kantis \& Federico, 2020). A medida que el emprendimiento se ve cada vez más como una construcción multidimensional (Montiel \& Rodríguez, 2017a), este se amplía desde múltiples ángulos, por ejemplo, desde una perspectiva evolutiva.

Esto contrasta con el paradigma de equilibrio puntuado que conceptualiza el cambio como una alternancia entre largos períodos en los que las infraestructuras estables sólo permiten adaptaciones incrementales y breves períodos de agitación revolucionaria. Gersick (1991) compara modelos desde seis visiones, adultez, grupo y desarrollo organizacional, historia de la ciencia, evolución biológica y ciencia física para explicar el paradigma del equilibrio puntuado y mostrar su amplia aplicabilidad en los estudios organizativos y yuxtapuestos para generar nuevas preguntas de investigación sobre un cambio en los entornos organizativos: cómo se activa, cómo funcionan los sistemas durante esos períodos y cómo concluye. Pero la evolución nunca termina.

\subsection{Dimensión 2: El Emprendedor}

Breslin (2008) examina el enfoque evolutivo del estudio del emprendimiento tanto a nivel de la población (ecología poblacional de las organizaciones) como a nivel organizativo (opción estratégica).

Breslin afirma que la elección estratégica se ha desarrollado a lo largo de los años desde un enfoque generalizado hacia las características empresariales y enfoques que se centran en el proceso y el contexto, incluyendo la cognición y el aprendizaje empresarial. Si bien la investigación empresarial se ha centrado en el nivel del emprendedor y startup, la investigación que utiliza un enfoque evolutivo para desarrollar una teoría a nivel de creación de nuevas empresas ha sido limitada. Por lo tanto, si bien el enfoque evolutivo se ha utilizado para examinar las organizaciones, en general a nivel de la elección estratégica, la investigación debería centrarse en aplicar el enfoque para examinar la adaptación y el aprendizaje de las pequeñas empresas (empresa familiar y la participación).

Además, Breslin menciona que el enfoque evolutivo ofrece más que los enfoques basados en la cognición y el aprendizaje, porque permite realizar un análisis multinivel del nuevo proceso de creación de empresas, que abarca tanto a la ecología de la población (nivel de población) y la perspectiva de elección estratégica (nivel organizativo), como a las interacciones resultantes entre ambas jerarquías. Por definición, una visión multidimensional actúa como una sinergia (como el caos y teorías complejas), de los tres enfoques (y otros), ofreciendo una valiosa visión de todo el proceso evolutivo.

Si bien la ecología de la población y la elección estratégica ofrecen perspectivas interesantes sobre el emprendimiento, hay limitaciones asociadas con cada una de 
ellas. Breslin afirma que los ecologistas de la población no hablan acerca del papel de la acción individual, y solo se centran en el proceso de selección bajo una organización que sobrevive en un mercado, mientras que los enfoques de elección estratégica no hacen mención al papel de la evolución de la población.

Este proceso se puede aplicar, por ejemplo, al aprendizaje donde Breslin y Jones (2012) presentan una perspectiva evolutiva sobre el aprendizaje empresarial, que refiere a los procesos ecológicos fundamentales centrados en el desarrollo de componentes clave del conocimiento dentro de pequeñas empresas nacientes y en crecimiento, y la evolución organizacional y las perspectivas coevolutivas de varios niveles que capturan procesos ecológicos ocultos dentro de los procesos de la idea, la construcción de nichos (Figura 2), y la lucha multinivel por la supervivencia, donde las capacidades gerenciales en el equipo deben cambiar (evolución), evaluando las necesidades cambiantes de la empresa (alto y bajo crecimiento, diversificación estratégica), la capacidad de adaptación (experiencia de equipo, diversidad funcional), y la capacidad de ejecutar el cambio (propiedad de gerente y CEO, independencia de la junta, participación de CR (Boeker \& Wiltbank, 2005).

Figura 1. Proceso de construcción de nichos

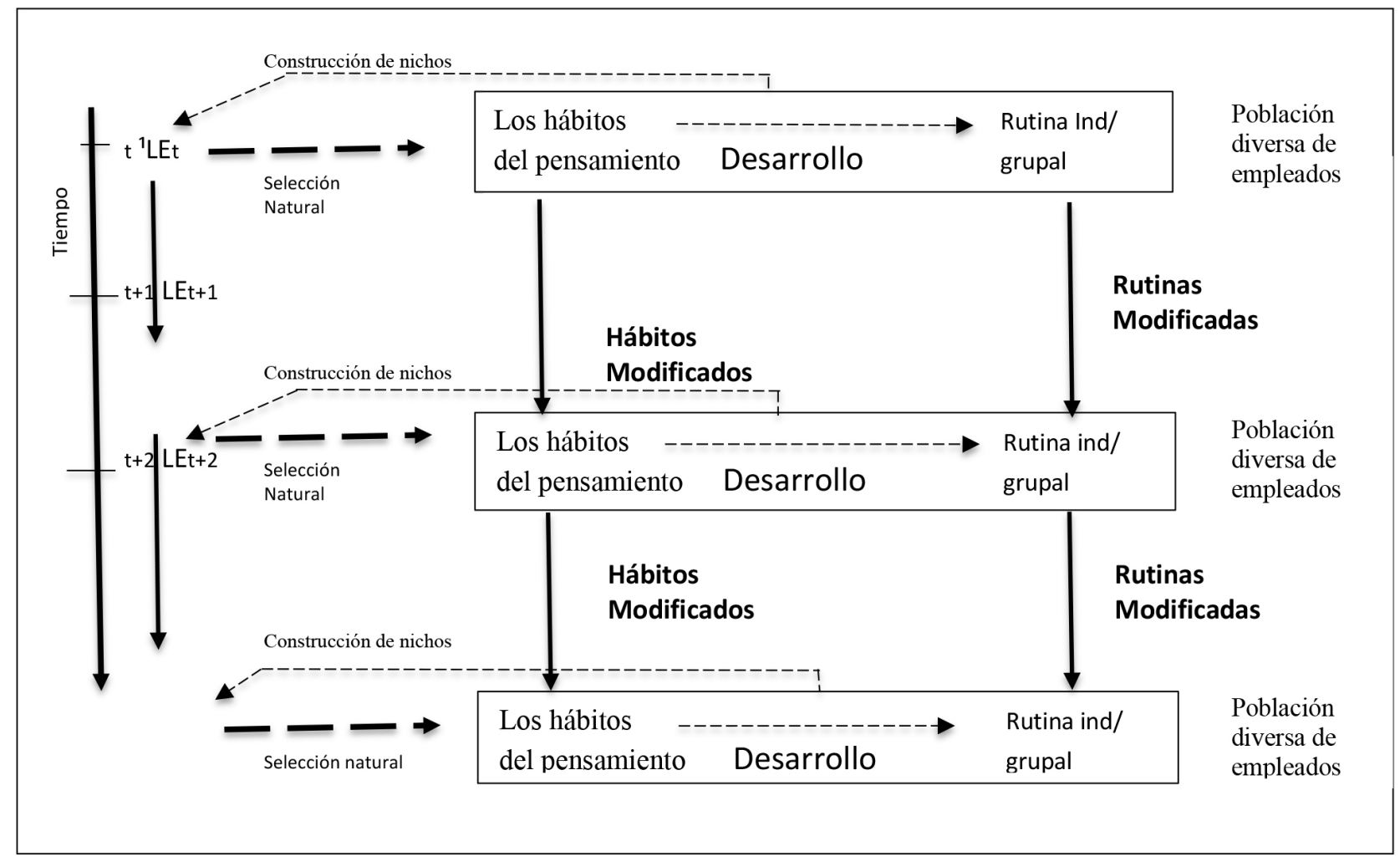

Fuente: Breslin y Jones (2012, p. 300), adaptada de Jones (2006).

Las competencias empresariales (Rasmussen et al., 2011) se basan en la perspectiva evolutiva, teniendo en cuenta la creación y el crecimiento temprano de cuatro spin-offs universitarias dentro del Reino Unido y Noruega, identificando tres competencias de oportunidad de perfeccionamiento, aprovechando y defendiendo lo que es crucial para que las empresas ganasen credibilidad, y aunque las competencias seleccionadas fuesen 
inherentes a los fundadores académicos, las competencias específicas para la creación de las empresas tuvieron que ser desarrolladas o adquiridas. Incluso al enfrentar el fracaso (Amankwah-Amoah et al., 2018), un emprendedor debe evolucionar para alcanzar el éxito en el futuro (por ejemplo, para aprender a ser resiliente).

La creación de una empresa podría ser el resultado del nivel de testosterona (White et al., 2006). Al igual que con la teoría psicológica evolutiva, el efecto biológico (nivel de testosterona) sobre el comportamiento (creación de nueva empresa) está parcialmente mediado por el psicológico (propensión al riesgo).

\subsection{Dimensión 3: Las empresas}

Las contribuciones de Campbell, especialmente para la teoría de la organización, fue un modelo de selección basado en la analogía entre la "selección natural en la evolución biológica y la propagación selectiva de formas culturales” (Campbell, 1965, p. 26). Campbell utilizó aspectos de su teoría de la selección para explicar la visión (1956a), la resolución de problemas (1956b), el pensamiento creativo (1960) y la evolución sociocultural $(1965,1979)$. Luego propuso dos antinomias que coexisten en las organizaciones: (1) obediencia a las rutinas culturales, normas y hábitos versus creatividad/experimentación y juego/fantasía; y (2) altruismo versus egoísmo. Desde el punto de vista de esta investigación, ambas pueden convertirse en una ventaja competitiva.

Los conceptos empresariales cumplen funciones importantes de manejo y motivación en la empresa (Witt, 1998). La interacción personal permite la difusión de conocimientos tácitos no verbales a través del aprendizaje observacional y la imitación de modelos a seguir. Un concepto empresarial compartido ayuda a consolidar las rutinas de las empresas, facilitando así la transferencia coordinada y la adaptación de las rutinas dentro de la empresa. El comprender e identificarse con los objetivos de la empresa aumenta la motivación intrínseca de los miembros de la misma y ayuda a mantener en control el comportamiento oportunista (Witt, 1998).

Según Bergstrom (2002), la selección del grupo mantiene el comportamiento cooperativo. Las fuerzas que apoyan este comportamiento incluyen la relación del grupo, la longevidad del grupo y las normas del grupo (la mayoría evoluciona en el tiempo para que este concepto empresarial siga siendo significativo para sus miembros y competitivo en el mercado).

La mayoría de las empresas a nivel mundial cuentan con una estructura familiar, la clave de la dimensión de la empresa es lo que Hodgson (2013) explica desde el enfoque darwiniano como "evolución” y "coevolución”, ampliamente utilizados en estudios de organización, pero que rara vez definen una estructura no clara si se refieren a entidades individuales o poblaciones. Por ejemplo, Hodgson afirma sobre los roles de adaptación individual y selección competitiva, y la posición "seleccionista” de Hannan y Freeman (1989), que hace hincapié en el papel de la selección y enfatiza los límites de la adaptabilidad de las empresas individuales que a menudo se describen como "Darwiniana", mientras que las opiniones opuestas que enfatizan la adaptabilidad se describen como "Lamarckiana". Los investigadores han demostrado que el principio darwiniano central, resultante de la comunidad ontológica abstracta en lugar de una analogía, se aplica a la evolución social para ayudar a entender la evolución de las organizaciones.

Así ha surgido un enfoque naturalista de la Teoría de la empresa, donde se ha explorado el papel de la cooperación y la evolución cultural (Cordes et al., 2008), y que 
menciona que hay una razón por la que existen las empresas, y es porque son organizaciones adecuadas dentro de las cuales pueden evolucionar los sistemas de producción cooperativos basados en las predisposiciones sociales humanas, y cómo un empresario, dadas estas predisposiciones, puede dar forma al comportamiento humano dentro de una empresa, ya que los seres humanos vivían en sistemas sociales a escala tribal basados en cantidades significativas de cooperación intragrupal e incluso intergrupal durante siglos, por lo que las empresas se basan en la psicología social que originalmente evolucionó para la vida tribal, pero las organizaciones modernas tienen funciones bastante diferentes de las tribus antiguas, lo que lleva a la fricción entre nuestras predisposiciones sociales y los objetivos de la organización, proponiendo que las empresas que logren reducir esta fricción tenderán a funcionar mejor.

Adicionalmente, Grabher y Stark (1997) examinan el carácter innovador que nace de la necesidad en el que los actores de entornos postsocialistas reestructuran, redefinen y recombinan los recursos, sin percibir estas combinaciones como accidentes sino más bien como un potencial evolutivo, donde la realidad del emprendimiento no es la personalidad individual aislada sino las redes sociales (sistema tribal) que vinculan a las empresas y a los actores dentro de ellas, debido al contexto donde se sustenta la escasez de capital y apoyo gubernamental/ecosistema. Las redes son clave para las startups desde una perspectiva longitudinal. Schutjens y Stam (2003) describen la evolución de las redes durante los tres primeros años después de la puesta en marcha y plantean explicar la naturaleza de las empresas jóvenes después de ese período.

Las empresas familiares están en constante evolución como campo de investigación (Bird et al., 2002). Zellweger y Sieger (2012), aplican orientación empresarial (OE) a empresas familiares con larga data, mostrando que un alto nivel para cada una de las cinco dimensiones $\mathrm{OE}$ no son necesarias para lograr el éxito a largo tiempo. Más bien, afirman que el nivel de OE se adapta con el tiempo, lo que implica entonces que esta construcción debe tener una evolución, en lugar de simplemente seguir creciendo.

Esta perspectiva evolutiva también se puede aplicar sobre el tamaño de las empresas (y de las organizaciones en general, como las universidades; Montiel \& Rodríguez, 2017b), donde investigaciones recientes sugieren que las empresas fundadas por individuos con experiencia previa en la misma empresa o similar (PFE relacionadas) tienden a sobrevivir más tiempo (Roberts et al., 2011), o cuando se alcanza el crecimiento de la empresa y existe un punto de reflexión donde se debe tomar una decisión sobre mantener al equipo fundador o traer uno con otro tipo de experiencia.

En cuanto a la evolución de la empresa familiar, Gersik et al. (1997) proponen el Modelo de Desarrollo Tridimensional, que considera la evolución independiente pero interconectada a tres subsistemas: propiedad, familia y empresa. Esta consideración es una contribución a la teoría general de los sistemas (Von Bertalanffy, 1940). Según el modelo de evolución, la dimensión de desarrollo de la propiedad se compone de tres etapas: control-propietario; sociedad y consorcio. La dimensión de desarrollo familiar se compone de cuatro etapas: negocios familiares; iniciación en el negocio; trabajo en equipo; y saber delegar. Por último, la dimensión de desarrollo empresarial se compone de tres etapas: puesta en marcha; expansión/formalización; y madurez. En este modelo, cada empresa familiar, según sus características, encuentra una etapa de 
cada subsistema. En la evolución, la empresa familiar pasará por períodos y desafíos críticos conocidos.

En relación a las transiciones empresariales, Breslin (2008) explora los factores que influyen al reemplazo del fundador, utilizando el emprendimiento y las teorías del ciclo de vida de la empresa, ya que las nuevas empresas pueden superar las capacidades gerenciales de sus equipos fundadores, y donde los fundadores pueden ser reemplazados por gerentes profesionales (Hannan \& Freeman, 1984, lo mencionan como un cambio también de la inercia estructural y organizacional). Además, existe un creciente interés en aplicar la psicología evolutiva en los negocios familiares, donde Nicholson (2008) propone que habrá una contribución única a ese campo, esbozando el marco darwiniano y sus implicaciones en un análisis de la dinámica del parentesco, la identidad de la propiedad y la transmisión intergeneracional, que es una herencia fundamental para entender las raíces de cooperación y conflicto en la empresa familiar.

En este tipo de empresa, la sucesión es un obstáculo clave para su viabilidad. Desde el enfoque de la economía evolutiva, Kansikas y Kuhmonen (2008) analizan la continuidad de la empresa familiar desde la generación fundadora hasta la 2 da generación. La sucesión de la empresa familiar y el pensamiento evolutivo en el cambio organizativo y económico se evalúan y combinan para proporcionar información con el fin de comprender la naturaleza de la sucesión de los negocios familiares, y se ilustra el funcionamiento de las fuerzas evolutivas clave - la variación, selección, retención y lucha- en la sucesión de las empresas familiares.

En cuanto a la variación, los autores buscan entender la importancia de contar con suficiente diversidad dentro de la empresa familiar, ya que esta diversidad de rutinas y competencias comprende el conjunto de variaciones a partir de la cual se puede hacer una selección cuando el entorno cambia. Con respecto a la selección, se debe entender el riesgo de sesgo de selección que está fácilmente arraigado a la cultura de la empresa familiar en relación a las relaciones familiares, emociones y valores, incluyendo decisiones sobre quién tendrá éxito y quién será el propietario de la empresa en el futuro.

\section{Hallazgos: Modelo propuesto}

La figura 3 es el modelo propuesto que sigue el criterio de Whetten (1989) sobre lo que constituye una contribución teórica. El modelo incluye factores considerados como parte de la explicación de la perspectiva evolutiva, siguiendo los criterios de amplitud (es decir, ¿están incluidos todos los factores relevantes?) para juzgar en qué medida se han incluido los factores "correctos". Además, indica cómo se relacionan (operativamente, utilizando "líneas" para conectar las "casilla", mostrando explícitamente patrones y causalidad (en el modelo se presenta una relación constante iterativa entre todos sus elementos). A continuación, el modelo, basado en la revisión de la literatura muestra la dinámica psicológica, económica o social subyacente (contexto multidimensional), y los factores en los entornos sociales y económicos que alteran y ejercen presión a la evolución/involución en el modelo y que justifican la selección de factores y las relaciones causales propuestas y su lógica. 
Figura 2. Marco exploratorio sobre el EEE, desde una perspectiva multidimensional sistémica evolutiva

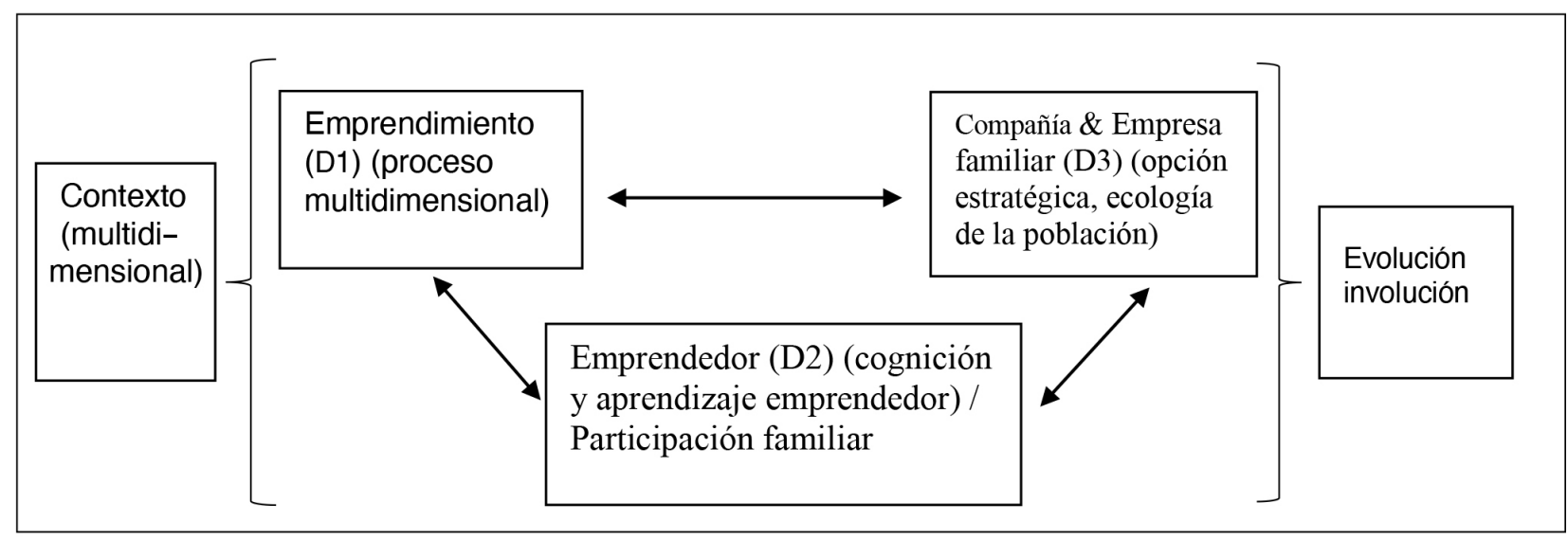

Fuente: Elaboración propia

Este modelo inicial tiene como propósito integrar las discusiones y hallazgos en una visión más cohesionada, por lo que podría ser un punto de partida para ver cómo la perspectiva evolutiva afecta a los elementos básicos de un sistema económico visto desde el emprendimiento. Después de Whetten, el propósito de un artículo teórico debe ser alterar la práctica de investigación. Nuestro modelo incorpora otros elementos aparte de los de elección estratégica y ecología poblacional sugeridos por Breslin (2008) en esta perspectiva evolutiva, comenzando con una visión más sistémica de la misma, incorporando el énfasis multidimensional en el contexto y el emprendimiento como un proceso (y como una construcción teórica), también añadiendo la participación familiar como un factor clave que influye en la evolución del empresario. Existe mucha literatura acerca de las empresas familiares y cómo difieren de las no familiares, y cómo se gestionan de manera diferente debido a los contextos, afectando así su funcionamiento y adaptación (evolución), o incluso la involución (cuando una empresa se niega a crecer, prefiriendo no correr riesgos, y se sostiene en la idea de mantener una pequeña empresa y producción sin sacrificar al personal).

Bajo este modelo se propone una visión sistémica, un enfoque multidimensional más integrado para analizar empíricamente la perspectiva evolutiva en los elementos que la constituyen, abriendo debates sobre el papel de la evolución en cada elemento.

\section{Conclusiones y discusión}

Existen diferencias entre la evolución social biológica y humana en términos de la dimensión conceptual de este último, la dependencia de la selección artificial y la necesidad de incorporar nociones de poder social como fuerza en la selección artificial y la herencia, entre otras. Tanto la evolución social biológica como la humana comparten una base cada vez más articulada al evolucionismo generalizado, que no es puramente Darwiniano ni puramente Lamarckiano.

Las organizaciones no pueden estar en un estado de histéresis. A lo largo de la investigación se ha presentado una breve reseña de los avances realizados hacia un estudio evolutivo integrado de emprendimiento, emprendedor y empresa, enfocado 
más en sus acciones y resultados, pero sin olvidar el incremento sobre los estudios basados en el emprendedor. En ese sentido, una mejor comprensión de la misma puede hacer una gran diferencia.

Al igual que Aldrich y Martínez (2007), una visión integrada del contexto, el proceso y los resultados de las actividades empresariales requiere pruebas empíricas más complejas. Hace años, era relativamente fácil probar hipótesis en este campo, y el éxito empresarial "dependía" de variables relativamente simples y estáticas.

Siguiendo un enfoque evolutivo (y los estudios de complejidad), el siguiente paso en la investigación empresarial debe ser la integración de métodos mixtos, para lograr una mejor comprensión de este hecho social. Además, también es necesario adoptar métodos mixtos integradores con áreas de investigación evolutiva, como la antropología evolutiva y la psicología evolutiva, a escuelas de negocios, institutos de estudios de innovación, y otros lugares.

En la misma perspectiva del juego de la evolución de la cultura génica, la teoría de la herencia dual puede ayudar a explicar el mecanismo que sustenta la herencia de comportamientos que involucran el conocimiento, las creencias e interacciones con otros individuos, pues esta actividad empresarial, aunque sea hereditaria, estará altamente supeditada a las fuerzas ecologistas.

\section{Referencias}

Aldrich, H., \& Martinez, M. (2007). Many are Called, but Few are Chosen: An Evolutionary Perspective for the Study of Entrepreneurship. In Álvaro Cuervo, Domingo Ribeiro, Salvador Roig (Eds.), Entrepreneurship, Concepts, Theory, and Perspective (pp. 293-312). Wiley.

Aldrich, H.E., Hodgson, G.M., Hull, D.L., Knudsen, T., Mokyr, J., \& Vanberg, V.J. (2008). In defense of generalized Darwinism. Journal of Evolutionary Economics, 18(5), 577-596. https://doi.org/10.1007/s00191-008-0110-z

Amankwah-Amoah, J., Boso, N., \& Antwi-Agyei, I. (2018). The Effects of Business Failure Experience on Successive Entrepreneurial Engagements: An Evolutionary Phase Model. Group $\mathcal{E}$ Organization Management, 43(4), 648-682. https://doi.org/10.1177/1059601116643447

Atran, S., Medin, D., \& Ross, N. (2004). Evolution and devolution of knowledge: A tale of two biologies. Journal of the Royal Anthropological Institute, 10(2), 395-420.

https://doi.org/10.1111/j.1467-9655.2004.00195.x

Berger, E., \& Kuckertz, A. (2016). Complexity in Entrepreneurship, Innovation and Technology Research, Applications of Emergent and Neglected Methods. Springer.

Bergstrom, T.C. (2002). Evolution of social behavior: individual and group selection. Journal of Economic Perspectives, 16(2), 67-88. https://doi.org/10.1257/0895330027265

Bird, B., Welsch, H., Astrachan, J.H., \& Pistrui, D. (2002). Family Business Research: The Evolution of an Academic Field. Family Business Review, 15(4), 337-350. https://doi.org/10.1111/j.1741-6248.2002.00337.x

Breslin, D. (2008), A review of the evolutionary approach to the study of entrepreneurship. International Journal of Management Reviews, 10(4), 399-423. doi:10.1111/j.1468-2370.2008.00234.x

Breslin, D., \& Jones, C. (2012). The evolution of entrepreneurial learning. International Journal of Organizational Analysis, 20(3), 294-308.

Boeker, W., \& Wiltbank, R. (2005). New Venture Evolution and Managerial Capabilities. Organization Science, 16(2), 123-133. https://bit.ly/3kth6F3

Bygrave, W., \& Hofer, C. (1991). Theorizing about Entrepreneurship. Entrepreneurship, Theory and Practice, 16(2), 13-22. https://doi.org/10.1177/104225879201600203

Campbell, D.T. (1956a). Perception as Substitute Trial and Error. Psychological Review, 63(5), 330-42. 
Campbell, D.T. (1956b). Adaptive Behavior from Random Response. Behavioral Science, 1(2), 105-110.

Campbell, D.T. (1960). Blind Variation and Selective Retention in Creative Thought as in Other Knowledge Processes. Psychological Review, 67(6), 380-400.

Campbell, D.T. (1965). Variation and Selective Retention in Socio-Cultural Evolution. In H.R. Barringer, G.I. Blankstein \& R.W. Mack (Eds.), Social Change in Developing Areas: A Reinterpretation of Evolutionary Theory. Schenkman.

Campbell, D.T. (1979). A tribal model of the social system vehicle carrying scientific knowledge. Knowledge: Creation, Diffusion, Utilization, 1(1), 181-201.

Carlsson, B., Braunerhjelm, P., McKelvey, M., Olofsson, C., Persson, L., \& Ylinenpa, H. (2013). The evolving domain of entrepreneurship research. Small Business Economics, 41, 913-930. http://doi. org/10.1007/s11187-013-9503-y

Cordes, C., Richerson, P.J., McElreath, R., \& Strimling, P. (2008). A naturalistic approach to the theory of the firm: the role of cooperation and cultural evolution. Papers on economics and evolution. Journal of Economic Behavior E Organization, 68(1), 125-139.

Freeman C. (1991). Innovation, Changes of Techno-Economic Paradigm and Biological Analogies in Economics. Revue Économique, 42(2), 211-232. https://bit.ly/31EKoYF

Grabher, G., \& Stark, D. (1997). Organizing Diversity: Evolutionary Theory, Network Analysis and Post socialism, Regional Studies, 31(5), 533-544. http://doi.org/10.1080/00343409750132315

Gersick, C.J. (1991). Revolutionary Change Theories: A Multilevel Exploration of the Punctuated Equilibrium. The Academy of Management Review, 16(1), 10-36. https://bit.ly/2XNyXMY

Gersick, K.E., Davis, J.A., McCollum, M., \& Lansberg, I. (1997). Generation to generation: Life cycles of the family business. Harvard Business Press. https:// doi.org/10.5860/choice.34-4568

Hannan, M.T., \& Freeman, J. (1984). Structural Inertia and Organizational Change, American Sociological Review, 49(2), 149-164.

Hannan, M.T., \& Freeman, J. (1989) Organizational Ecology. Harvard University Press.

Hodgson, G.M. (2013). Understanding Organizational Evolution: Toward a Research Agenda using Generalized Darwinism. Organization Studies, 34(7), 973-992. http://doi. org $/ 10.1177 / 0170840613485855$

Jackson, D. J. (2011). What is an Innovation Ecosystem, National Science Foundation, Arlington, VA. 1-11. https://bit.ly/2PKyoz7

Kantis, H., \& Federico, J. (2020). A dynamic model of entrepreneurial ecosystems evolution. Journal of Evolutionary Studies in Business, 5(1), 182-220.

Kansikas, J., \& Kuhmonen. T. (2008). Family Business Succession: Evolutionary Economics Approach. Journal of Enterprising Culture, 16(3), 279-298.

Landström, H. (2020). The Evolution of Entrepreneurship as a Scholarly Field. Foundations and Trends ${ }^{\circledR}$ in Entrepreneurship, 16(2), 65-243. http://doi.org/10.1561/0300000083

Lichtenstein, B.B. (2000). Emergence as a process of self-organizing. New assumptions and insights from the study of non-linear dynamic systems. Journal of Organizational Change Management, 13(6), 526-544. https://bit.ly/2YSUaFV

Madsen, T.K., \& Servais, P. (1997). The Internationalization of Born Globals: an Evolutionary Process? International Business Review, 6(6), 561-583.

https://doi.org/10.1016/S0969-5931(97)00032-2

Malecki, E. J. (2011). Connecting local entrepreneurial ecosystems to global innovation networks: Open innovation, double networks and knowledge integration, International Journal of Entrepreneurship and Innovation Management, 14(1), 36-59.

http://doi.org/10.1504/ijeim.2011.040821

Metcalfe, J. S. (2006). Entrepreneurship: An evolutionary perspective. In The Oxford Handbook of Entrepreneurship. http://doi.org/10.1093/oxfordhb/9780199546992.003.0003

Meredith, A. (1982). Devolution. Journal of Theoretical Biology, 96(1), 49-65. http://doi.org/10.1016/0022-5193(81)90155-2

Mitchell, M. (2009). Complexity: A Guided Tour. Oxford University Press.

Montiel, O., \& Rodríguez, C. (2017a). Emprendimiento Hoy. Multidimensionalidad, Cambio E Innovación. 
Universidad Autónoma de Ciudad Juárez.

Montiel, O., \& Rodríguez, C. (2017b). Would I do it again? Revisiting the implementation of the entrepreneurship paradigm in a University in Mexico. Retos, Revista de Ciencias de la Administración y Economía, 7(14), 147-165. https://doi.org/10.17163/ret.n14.2017.08

Moore, B.S., \& Manring, S.L. (2009). Strategy development in small and medium-sized enterprises for sustainability and increased value creation, Journal of Cleaner Production, 17(2), 276-282. http://doi.org/10.1016/j.jclepro.2008.06.004

Nicholson, N. (2008). Evolutionary Psychology and Family Business: A New Synthesis for Theory, Research, and Practice. Family Business Review, 21(1), 103-118. http://doi.org/10.1111/j.1741-6248.2007.00111.x

Rasmussen, E., Mosey, S., \& Wright, M. (2011). The Evolution of Entrepreneurial Competencies: A Longitudinal Study of University Spin-Off Venture Emergence. Journal of Management Studies, 48(6), 1314-1345. http://doi.org/10.1111/j.1467-6486.2010.00995.x

Roberts, P.W., Klepper, S., \& Hayward, S. (2011). Founder backgrounds and the evolution of firm size. Industrial and Corporate Change, 20(6), 1515-1538. Oxford University Press.

Schutjens, V., \& Stam, E. (2003). The Evolution and Nature of Young Firm Networks: a Longitudinal Perspective, Small Business Economics, 21(2), 115-134.

$\mathrm{Su}$, Z. (2020). The co-evolution of institutions and entrepreneurship. Asia Pacific Journal of Management. http://doi.org/10.1007/s10490-019-09703-y

Veciana, J.M. (2007). Entrepreneurship as a Scientific Research Programme. In Álvaro Cuervo, Domingo Ribeiro, Salvador Roig (Eds.), Entrepreneurship, Concepts, Theory and Perspective (pp. 23-72). Wiley.

Von Bertalanffy, L. (1940). Der Organismus als physikalisches System betrachtet. Naturwissenschaften, 28(33), 521-531. http://doi.org/10.1007/bf01497764

Whetten, D.A. (1989). What Constitutes A Theoretical Contribution? Academy of Management Review, 14(4), 490-495. http://doi.org/10.2307/258554

White, R.E, Thornhill, S., \& Hampson, E. (2006). Entrepreneurs and evolutionary biology: The relationship between testosterone and new venture creation, Organizational Behavior and Human Decision Processes, 100(1), 21-34. http://doi.org/10.1016/j.obhdp.2005.11.001.

Witt, U. (1998). Imagination and leadership. the neglected dimension of an evolutionary theory of the firm, Journal of Economic Behavior and Organization, 35(2), 161-177. http://doi.org/10.1016/ s0167-2681(98)00058-4

Wolff, P., Medin, D.L., \& Pankratz, C. (1999). Evolution and devolution of folkbiological knowledge. Cognition, 73(2), 177-204. http://doi.org/10.1016/S0010-0277(99)00051-7

Zellweger, T., \& Sieger, P. (2012). Entrepreneurial orientation in long-lived family firms. Small Business Economics, 38(1), 67-84. http://doi.org/10.1007/s11187-010-9267-6 